\title{
Comparación entre las jornadas escolares y el resultado de las pruebas Saber 2017, en el municipio de Soacha, Cundinamarca
}

\author{
Jorge Enrique Díaz-Pinzón ${ }^{1}$
}

Recibido: 09-05-2019

Aceptado: $21-08-2019$

\section{Resumen}

Se realizó un estudio de investigación relacionado con los puntajes obtenidos por los estudiantes de grado once de las diferentes instituciones educativas oficiales del municipio Soacha, Cundinamarca. A partir del análisis estadístico de los datos con el uso de Anova, se observó que el p-valor de la prueba es igual a 0,00 para el promedio de la prueba Saber 11 2017, para lectura crítica; 0,00 para matemáticas; 0,00 para sociales; 0,00 para ciencias naturales y 0,00 para inglés, resultados que son menores a $\alpha=$ 0,05 , de manera que se rechaza Ho, es decir que hay diferencias significativas entre los promedios. Las instituciones educativas con mejor promedio de la prueba Saber 112017 por jornada fueron las siguientes: Santa Ana jornada mañana, con 55,86; Julio César Turbay Ayala - Sede principal jornada mañana, con 53,77. Se evidencia un mejor resultado en las jornadas de la mañana sobre las jornadas tarde.

Palabras clave: educación, calidad de la educación, evaluación comparativa, educación comparada.

\footnotetext{
1. Magíster en Gestión de la Tecnología Educativa; docente de Matemáticas, I. E. General Santander. Soacha (Cundinamarca, Colombia).

Correo electrónico: jediazp@unal.edu.co, jorgediaz333@gmail.com

ORCID: https://orcid.org/0000-0002-8870-7769

Google Scholar: https://scholar.google.es/citations?user=f4PqfaoAAAAJ\&hl=es
} 


\section{Comparison between school sessions (morning or afternoon) and the result of the Saber 2017 tests in the municipality of Soacha, Cundinamarca}

\section{Abstract}

A study was carried out related to the scores obtained by eleventh grade students of the different public educational institutions of the municipality of Soacha, Cundinamarca. The statistical analysis of the data with the use of Anova show that the $p$-value of the test is equal to 0.00 for the average of the Saber 112017 test for critical reading; 0.00 for math; 0.00 for social; 0.00 for natural sciences and 0.00 for English. These results are less than $\alpha=0.05$, so $\mathrm{Ho}$ is rejected, meaning that there are significant differences between the averages. The educational institutions with the best average of the Saber 112017 test by session are the following: Santa Ana morning session, with 55.86; Julio César Turbay Ayala main campus morning session, with 53.77. The morning sessions show better results than the afternoon sessions.

Keywords: education, quality of education, comparative $\neg$ evaluation, compared education.

\section{Introducción}

Según el Congreso de la República de Colombia (2009), la Ley 1324 de 2009 le confiere al Instituto Colombiano para Evaluación de la Educación (Icfes) la misión de evaluar, mediante exámenes externos estandarizados, la formación que se ofrece en el servicio 
educativo en los distintos niveles. También establece que el Ministerio de Educación Nacional (MEN) debe definir lo que se evalúa en estos exámenes.

Según el Ministerio de Educación Nacional (MEN, 2010), el Examen de Estado de la educación media Saber $11 .^{\circ}$ evalúa el nivel de la Educación Media y, a partir del 2014, se ordena con las evaluaciones de la Educación Básica para suministrar información a la comunidad educativa sobre el progreso de las competencias básicas que debe desarrollar un estudiante durante el paso por la vida escolar. Por tal motivo, deber ser un instrumento que retroalimenta al Sistema Educativo.

Todos los estudiantes que se encuentren ultimando el grado undécimo deben ser evaluados por medio de este instrumento, con el fin de obtener resultados oficiales para efectos de admisión a la educación superior. También pueden presentarlo quienes ya hayan obtenido el título de bachiller o hayan superado el examen de validación del bachillerato, de anuencia con las disposiciones vigentes (Icfes, 2017).

Actualmente, el MEN entiende el objetivo de la educación como el desarrollo de determinadas competencias $y$, en efecto, a estas como el objeto de la evaluación. Dentro de las disímiles competencias que pueden desplegarse a lo largo del proceso educativo, se diferencia entre las "genéricas" y las "no genéricas". Las primeras son aquellas que resultan indefectibles para el desempeño social, laboral y cívico de todo ciudadano, aparte de su oficio o profesión. Las segundas son aquellas propias de disciplinas específicas, que resultan indefectibles para profesiones u oficios concretos (Icfes, 2017).

Según el Icfes (2017), en el examen se esgrimen preguntas de selección múltiple con única respuesta. Estas están constituidas por un enunciado (que presenta una situación, contexto, texto, etc.), la formulación de una tarea de evaluación (aquello que se le pide al estudiante realizar) y cuatro alternativas de respuesta, agrupadas como A, B, C y D, de las cuales solo una es correcta o válida dada 
la tarea planteada. El estudiante debe elegir entre estas opciones la que considere apropiada.

Existen varios trabajos de investigación al respecto en Colombia, con diversas conclusiones. Gaviria y Barrientos (2001) han dedicado sus investigaciones a estudiar los determinantes del rendimiento académico en nuestro país, y consideran algunos determinantes del rendimiento académico de los estudiantes en Bogotá, de cuyo estudio se desglosan tres conclusiones principales: 1) la educación de los padres tiene un resultado sustancial sobre el rendimiento académico; 2) el efecto de la educación de los padres se divulga especialmente a través de la calidad de los planteles educativos, y 3) los planteles inciden de manera transcendental sobre el rendimiento en esta ciudad.

Según Sánchez y Muñoz (2014), una de las grandes dificultades que se presentan en los estudiantes es el escaso interés y estimulación frente a las trabajos escolares, que redunda en una exigua comprensión lectora en las disímiles áreas del conocimiento y la poca exegesis que se hace mucho más notoria especialmente en la solución de problemas matemáticos. Esto hace que no se obtengan los resultados deseados en las pruebas saber para las Instituciones. Por esa razón, el objetivo de este trabajo fue comparar los resultados obtenidos en las jornadas mañana y tarde, a partir del análisis estadístico de los datos aplicando un análisis de varianza (Anova).

\section{Metodología}

El método de investigación utilizado fue el cuantitativo. Según Shuttleworth (2008), todos los experimentos cuantitativos esgrimen un formato estándar, con algunos pequeños contrastes interdisciplinarios para formar una hipótesis que será probada o desmentida. Esta hipótesis debe ser justificable por medios matemáticos y estadísticos y establece la base alrededor de la cual se delinea todo el experimento. 


\section{Población}

Esta investigación se centró en las 56 jornadas de las diferentes instituciones educativas oficiales del municipio de Soacha, Cundinamarca, con la información de los resultados de la prueba Saber 11. ${ }^{\circ}-2$ para el 2017.

\section{Resultados}

\section{Hipótesis con el Anova}

La hipótesis del presente trabajo de investigación se diseña como una relación causal y se enuncia de la siguiente forma:

Hipótesis alterna (Ha): Hay diferencias en el promedio de los puntajes obtenidos en lectura crítica, matemáticas, ciencias sociales, ciencias naturales e inglés de la prueba Saber $11 .^{\circ}$ entre las 56 jornadas de las diferentes instituciones educativas oficiales del municipio de Soacha, Cundinamarca, con base en la información de los resultados de la prueba Saber $11 .^{\circ}-2$ para el 2017.

Hipótesis nula (Ho): No hay diferencias en el promedio de los puntajes obtenidos en lectura crítica, matemáticas, ciencias sociales, ciencias naturales e inglés de la prueba Saber $11 .^{\circ}$ entre las 56 jornadas de las diferentes instituciones educativas oficiales del municipio de Soacha-Cundinamarca, Colombia, con base en la información de los resultados de la prueba Saber $11 .^{\circ}-2$ para el 2017. 


\section{Prueba estadística}

El valor de significancia de la prueba es de $\alpha=0,05$ (5\%). Si el valor es mayor, se acepta la hipótesis nula; si es menor, se rechaza la hipótesis nula. Para la comparación entre medias independientes, se utilizó el software SPSS v. 20.0.

En la tabla 1, se observa que el $p$-valor de la prueba es igual a 0,00 para el promedio de la prueba Saber $11 .^{\circ}$ para lectura crítica es 0,00; para matemáticas, 0,00; para sociales, 0,00; para ciencias naturales, 0,00, y para inglés, 0,00 . Todos los valores son menores a $\alpha=0,05$, de manera que se rechaza Ho; es decir, hay diferencias significativas entre los promedios.

Tabla 1. Anova de un factor para el promedio de puntaje de la prueba Saber $11 .^{\circ}-2$ del 2017.

\begin{tabular}{|c|c|c|c|c|c|c|}
\hline & & $\begin{array}{l}\text { Suma de } \\
\text { cuadrados }\end{array}$ & gl & $\begin{array}{l}\text { Media } \\
\text { cuadrática }\end{array}$ & $\mathbf{F}$ & Sig. \\
\hline \multirow{3}{*}{ Promedio } & Inter-grupos & 427,377 & 4 & 106,844 & 21,810 & 0,000 \\
\hline & Intra-grupos & 249,846 & 51 & 4,899 & & \\
\hline & Total & 677,223 & 55 & & & \\
\hline \multirow{3}{*}{ Lectura Crítica } & Inter-grupos & 287,982 & 4 & 71,995 & 12,758 & 0,000 \\
\hline & Intra-grupos & 287,812 & 51 & 5,643 & & \\
\hline & Total & 575,793 & 55 & & & \\
\hline \multirow{3}{*}{ Matemáticas } & Inter-grupos & 706,433 & 4 & 176,608 & 22,889 & 0,000 \\
\hline & Intra-grupos & 393,515 & 51 & 7,716 & & \\
\hline & Total & 1099,947 & 55 & & & \\
\hline \multirow{3}{*}{ Sociales } & Inter-grupos & 286,934 & 4 & 71,733 & 11,031 & 0,000 \\
\hline & Intra-grupos & 331,645 & 51 & 6,503 & & \\
\hline & Total & 618,579 & 55 & & & \\
\hline \multirow{3}{*}{ Ciencias } & Inter-grupos & 517,721 & 4 & 129,430 & 32,106 & 0,000 \\
\hline & Intra-grupos & 205,599 & 51 & 4,031 & & \\
\hline & Total & 723,319 & 55 & & & \\
\hline \multirow{3}{*}{ Inglés } & Inter-grupos & 445,734 & 4 & 111,434 & 23,095 & 0,000 \\
\hline & Intra-grupos & 246,071 & 51 & 4,825 & & \\
\hline & & 691,806 & 55 & & & \\
\hline
\end{tabular}

Fuente: elaboración propia. 
Ahora bien, cuando Ho se rechaza en la prueba Anova, entonces se procede a realizar una prueba Post Hoc. En este caso, la prueba de Duncan, que es un test de comparaciones múltiples, admite comparar las medias de los t niveles de un factor, luego de haber rechazado la hipótesis nula de igualdad de medias mediante la técnica Anova (Llopis, 2013), en muestras independientes; asimismo, permite observar si hay diferencias entre cada uno de los niveles, como se puede observar en las tablas $2,3,4,5,6$ y 7 . En todas las tablas se aprecian dos subconjuntos con un $\alpha=0,05$.

Tabla 2. Prueba de Duncan-Promedio.

\begin{tabular}{|c|c|l|l|}
\hline \multirow{2}{*}{ Jornada } & \multirow{2}{*}{ N } & \multicolumn{2}{|l|}{$\begin{array}{l}\text { Subconjunto para } \\
\mathbf{\alpha}=\mathbf{0 , 0 5}\end{array}$} \\
\cline { 3 - 4 } & & $\mathbf{1}$ & $\mathbf{2}$ \\
\hline Fin de semana & 15 & 44,6887 & \\
\hline Nocturna & 4 & 45,7275 & \\
\hline Tarde & 9 & & 50,4456 \\
\hline Mañana & 24 & & 50,7979 \\
\hline Única & 4 & & 50,8050 \\
\hline Sig. & & 0,386 & 0,778 \\
\hline
\end{tabular}

Fuente: elaboración propia.

Tabla 3. Prueba de Duncan-Matemáticas.

\begin{tabular}{|c|c|c|c|}
\hline \multirow{2}{*}{ Jornada } & \multirow{2}{*}{ N } & \multicolumn{2}{|c|}{$\begin{array}{l}\text { Subconjunto para } \\
\mathbf{\alpha}=\mathbf{0 , 0 5}\end{array}$} \\
\cline { 3 - 4 } & & $\mathbf{1}$ & $\mathbf{2}$ \\
\hline Fin de semana & 15 & 42,0220 & \\
\hline Nocturna & 4 & 42,8300 & \\
\hline Tarde & 9 & & 49,1056 \\
\hline Mañana & 24 & & 49,7388 \\
\hline Única & 4 & & 50,3950 \\
\hline Sig. & & 0,590 & 0,421 \\
\hline
\end{tabular}

Fuente: elaboración propia. 
Tabla 4. Prueba de Duncan-Lectura crítica.

\begin{tabular}{|c|c|l|l|}
\hline \multirow{2}{*}{ Jornada } & \multirow{3}{*}{ N } & \multicolumn{2}{|l|}{$\begin{array}{l}\text { Subconjunto para } \\
\text { N }=\mathbf{0 , 0 5}\end{array}$} \\
\cline { 2 - 4 } & & 1 & 2 \\
\hline Fin de semana & 15 & 48,1413 & \\
\hline Nocturna & 4 & 48,6525 & \\
\hline Única & 4 & & 51,4225 \\
\hline Tarde & 9 & & 52,6878 \\
\hline Mañana & 24 & & 53,2683 \\
\hline Sig. & & 0,690 & 0,178 \\
\hline
\end{tabular}

Fuente: elaboración propia.

Tabla 5. Prueba de Duncan-Sociales.

\begin{tabular}{|c|c|l|l|}
\hline \multirow{2}{*}{ Jornada } & \multirow{2}{*}{ N } & \multicolumn{2}{|l|}{$\begin{array}{l}\text { Subconjunto para } \\
\text { a }=0,05\end{array}$} \\
\cline { 2 - 4 } & & 1 & 2 \\
\hline Fin de semana & 15 & 44,7553 & \\
\hline Nocturna & 4 & 45,5325 & \\
\hline Tarde & 9 & & 49,2678 \\
\hline Mañana & 24 & & 49,6233 \\
\hline Única & 4 & & 50,6000 \\
\hline Sig. & & 0,572 & 0,365 \\
\hline
\end{tabular}

Fuente: elaboración propia.

Tabla 6. Prueba de Duncan-Ciencias.

\begin{tabular}{|c|c|l|l|}
\hline \multirow{2}{*}{ Jornada } & \multirow{3}{*}{ N } & \multicolumn{2}{|l|}{$\begin{array}{l}\text { Subconjunto para } \\
\alpha=\mathbf{0 , 0 5}\end{array}$} \\
\cline { 3 - 4 } & & 1 & 2 \\
\hline Fin de semana & 15 & 44,5860 & \\
\hline Nocturna & 4 & 46,4775 & \\
\hline Tarde & 9 & & 51,1889 \\
\hline Mañana & 24 & & 51,2758 \\
\hline Única & 4 & & 51,9275 \\
\hline Sig. & & 0,085 & 0,523 \\
\hline
\end{tabular}

Fuente: elaboración propia. 
Tabla 7. Prueba de Duncan-Inglés.

\begin{tabular}{|c|c|l|l|}
\hline \multirow{2}{*}{ Jornada } & \multirow{2}{*}{ N } & \multicolumn{2}{|l|}{$\begin{array}{l}\text { Subconjunto para } \\
\alpha=0,05\end{array}$} \\
\cline { 3 - 4 } & & 1 & 2 \\
\hline Fin de semana & 15 & 42,4493 & \\
\hline Nocturna & 4 & 43,9750 & \\
\hline Única & 4 & & 47,4450 \\
\hline Mañana & 24 & & 48,6787 \\
\hline Tarde & 9 & & 49,0333 \\
\hline Sig. & & 0,201 & 0,210 \\
\hline
\end{tabular}

Fuente: elaboración propia.

Tabla 8. Promedio de puntaje de la prueba Saber $11 .^{\circ}-2$ del 2017.

\begin{tabular}{|c|c|c|}
\hline Institución Educativa & Jornada & Promedio \\
\hline Ciudad Latina & Tarde & 48,6500 \\
\hline Nuevo Compartir & Tarde & 52,5850 \\
\hline Compartir & Única & 47,2450 \\
\hline Santa Ana & Mañana & 55,8600 \\
\hline Integrado de Soacha & Única & 48,9575 \\
\hline General Santander & Mañana & 53,2100 \\
\hline La Despensa & Mañana & 50,0100 \\
\hline Manuela Beltran & Nocturna & 49,0900 \\
\hline León XIII & Nocturna & 49,0267 \\
\hline Buenos Aires & Nocturna & 47,4267 \\
\hline Ciudadela Sucre & Mañana & 48,7100 \\
\hline Julio Cesar Turbay & Mañana & 53,7790 \\
\hline Luis Carlos Galán & Tarde & 45,2350 \\
\hline Gabriel García Márquez & Tarde & 45,0550 \\
\hline Cazucá & Tarde & 46,1400 \\
\hline El Bosque & Nocturna & 46,4667 \\
\hline San Mateo & Mañana & 52,8700 \\
\hline Ricaurte & Tarde & 46,3000 \\
\hline Las Villas & Tarde & 45,4000 \\
\hline Eduardo Santos & Mañana & 51,8700 \\
\hline Eugenio Díaz Castro & Mañana & 49,8200 \\
\hline Chiloé & Tarde & 53,0200 \\
\hline Ciudad Verde & Nocturna & 50,0267 \\
\hline Varon de Sol & Mañana & 52,3700 \\
\hline
\end{tabular}




\begin{tabular}{|c|c|c|}
\hline Institución Educativa & Jornada & 52,4700 \\
\hline $\begin{array}{c}\text { Institucion Educativa Nuevo Compartir } \\
\text { Sede B }\end{array}$ & Mañana & 51,4300 \\
\hline Colegio Benedicto XVI - Sede Principal & Mañana & 49,1600 \\
\hline $\begin{array}{c}\text { Institucion Educativa Eugenio Diaz } \\
\text { Castro Sede H }\end{array}$ & Mañana & 47,1750 \\
\hline $\begin{array}{c}\text { Institucion Educativa Ciudadela Sucre } \\
\text { Sede B }\end{array}$ & Mañana & 42,9800 \\
\hline $\begin{array}{c}\text { Institucion Educativa Eduardo Santos } \\
\text { Sede C }\end{array}$ & Mañana & \\
\hline
\end{tabular}

Fuente: elaboración propia.

En la tabla 8 se aprecia que las instituciones educativas del municipio de Soacha, Cundinamarca, con mejor promedio de la prueba Saber 112017 por jornada fueron Santa Ana jornada mañana, con 55,86; Julio César Turbay Ayala - Sede principal, jornada mañana, con 53,77; General Santander, jornada mañana, con 53, 21; Chiloé, jornada tarde, 53,02; y San Mateo, jornada mañana, con 52,87.

\section{Conclusiones}

A partir del análisis estadístico de los datos a través del Anova, se observó que el p-valor de la prueba es igual a 0,00 para el promedio de la prueba Saber $11 .^{\circ}$, así como en cada una de las materias en particular. Al ser estos valores menores a $\alpha=0,05$, se rechaza Ho, es decir que hay diferencias significativas entre los promedios.

Las instituciones educativas del municipio de Soacha, Cundinamarca, con mejor promedio de la prueba Saber 112017 por jornada fueron Santa Ana jornada mañana, con 55,86; Julio César Turbay Ayala - Sede principal, jornada mañana, con 53, 77; General Santander, jornada mañana, con 53, 21; Chiloé, jornada tarde, 53,02; Chiloé, jornada mañana, con 52,94, y San Mateo, jornada mañana, con 52,87. Se evidencia un mejor resultado en las jornadas de la mañana sobre las jornadas tarde. Se propone realizar un estudio más exhaustivo para determinar por qué las jornadas mañana tie- 
nen un mejor comportamiento en el resultado de las Prueba Saber 11 que las jornadas tarde.

Hay que implementar estrategias de mejoramiento para la prueba Saber 11. ${ }^{\circ}$; particularmente, se debe enfatizar en la planeación y aplicación de estas pruebas en las diferentes instituciones. Una de las maneras para hacerlo, es mejorando las prácticas de aula en lectura crítica, matemáticas, sociales, ciencias naturales e inglés. Díaz (2016a y b; 2017a y b) proporciona ejemplos de ello sobre el uso de simuladores, logrando evidenciar una mejora en la enseñanza de las matemáticas. Además, se debe tener una formación permanente de los docentes en el conocimiento y apropiación de las tecnologías de la información y la comunicación (Díaz, 2017c; 2017d).

Estos resultados se verán reflejados en el Índice Sintético de Calidad Educativa (ISCE) para el 2018, recordando que los mejores índices de básica primaria, básica secundaria y media fueron para Eugenio Díaz Castro con un promedio de 6,86, Santa Ana, con 6,16, y el General Santander, con 6,09 (Díaz, 2017e).

\section{Referencias bibliográficas}

Chica, S., Galvis, D., \& Ramírez, A. (2009). Determinantes del rendimiento académico en Colombia: pruebas ICFES Saber 11으. 2009. Revista Universidad EAFIT, 44(160), 48-72 Recuperado de http://publicaciones. eafit.edu.co/index.php/revista-universidad-eafit/article/view/754

Congreso de la República de Colombia. (2009). Ley 1324 de 2009: por la cual se fijan parámetros y criterios para organizar el sistema de evaluación de resultados de la calidad de la educación, se dictan normas para el fomento de una cultura de la evaluación, en procura de facilitar la inspección y vigilancia del Estado y se transforma el ICFES. Diario Oficial, 13 de julio de 2009, n. ${ }^{\circ}$ 47.409. Bogotá, D. C.: Congreso de la República. 
Cortés, A; García, G. (2017). Estrategias pedagógicas que favorecen el aprendizaje de niñas y niños de 0 a 6 años de edad en VillavicencioColombia. Revista Interamericana de Investigación, Educación y Pedagogía, RIIEP, 10(1). DOI: https://doi.org/10.15332/25005421

de Almeida, R. R., Santos, M. F., \& Porto, J. C. (2017). Lectura de textos ficcionales y el enfoque escolar de literatura: contribuciones para una Pedagogía de la Elección. Revista Interamericana de Educación, Pedagogía y Estudios Culturales, 9(1), 35-51. DOI: https://doi. org/10.22490/25391887.1925

Díaz, J. (2016a). Aplicación PhET: estrategia de enseñanza-aprendizaje de fracciones equivalentes. Revista Criterios, 23(1), 99-11. Recuperado de http://www.ojseditorialumariana.com/index.php/Criterios/article/ view/1789

Díaz, J. (2016b). Soporte técnico de simulación Phet en la enseñanza y aprendizaje de fracciones equivalentes. Revista de Investigaciones Universidad del Quindío, 28(2), 31-41. doi: http://doi.org/c89g

Díaz, J. (2017a). Importancia de la simulación Phet en la enseñanza y aprendizaje de fracciones equivalentes. Revista Educación y Desarrollo Social, 11(1), 48-63. doi: http://doi.org/c89f

Díaz, J. (2017b) Simulador Applet Descartes: Como didáctica de enseñanza de la función cuadrática. Innova Research Journal, 2(8), 6978. doi: http://doi.org/c89d

Díaz, J. (2017c). Conocimiento y apropiación de las tecnologías de la información y la comunicación (TIC) en la formación docente. Revista Actualidades Pedagógicas, 69, 121-131. doi: http://doi.org/c89c

Díaz, J. (2017d). Formación de docentes en el uso y apropiación de las Tic. Innova Research Journal, 2(9), 163-168. doi: http://doi.org/c89b

Díaz, J. (2017e). Valoración de los resultados del Índice Sintético de la calidad educativa (ISCE) 2017 de las instituciones oficiales en el 
municipio de Soacha-Cundinamarca. Innova Research Journal, 2(11), 126-134. (2017). doi: http://doi.org/c889

Gaviria, A., \& Barrientos, J. (2001). Determinantes de la educación en Colombia. Planeación de Desarrollo, 32(3), 339-386. Recuperado de https://colaboracion.dnp.gov.co/CDT/RevistaPD/2001/pd_vXXXII_ n3_2001_art.1.pdf

Instituto Colombiano para la Evaluación de la Educación Superior (Icfes). (2017). Guía de orientación Saber 11. . Recuperado de www.icfes.gov. co/...y.../saber-11/...saber-11/...saber-11/...saber-11.../file?...

Langer, E. (2016). La construcción de confianza para el estudio de prácticas de resistencia en la escolarización de jóvenes en contextos de pobreza urbana. Revista Interamericana de Investigación, Educación y Pedagogía, RIIEP, 9 (2). DOI: https://doi.org/10.15332/25005421

Llopis, J. (2013). La estadística: una orquesta hecha instrumento. Recuperado de https://estadisticaorquestainstrumento.wordpress. com/2013/01/28/test-de-duncan/

Ministerio de Educación Nacional (MEN). (2010). Pruebas Saber. Recuperado de http:// www.mineducacion.gov.co/ 1759/ w3- article244735.html

Sánchez, L., \& Muñoz, N. (2014). Prepárate divertidamente para las pruebas saber, Tesis de grado. Universidad Católica de Manizales, Manizales, Colombia. Recuperado de http://repositorio.ucm.edu. co:8080/jspui/bitstream/handle/10839/994/Nancy\%20Liliana\%20 Munoz\%20Betancurth.pdf?sequence=1\&isAllowed=y

Shuttleworth, M. (2008). Diseño de la investigación cuantitativa. Recuperado de https://explorable.com/es/diseno-de-la-investigacioncuantitativa 